\title{
Translocalization of enhanced PKM2 protein into the nucleus induced by cancer upregulated gene 2 confers cancer stem cell-like phenotypes
}

\author{
Natpaphan Yawut ${ }^{1, \#}$, Sirichat Kaowinn, ${ }^{2, \#}$, Il-Rae Cho ${ }^{1}$, Phatcharaporn Budluang ${ }^{1}$, Seonghye Kim ${ }^{3}$, Suhkmann Kim ${ }^{3}$, \\ So Eun Youn ${ }^{4}$, Sang Seok Koh ${ }^{4}$ E Young-Hwa Chung ${ }^{1, *}$ \\ ${ }^{1}$ BK21 plus, Department of Cogno-Mechatronics Engineering, Optomechatronics Research Center, Busan 46241, Korea, ${ }^{2}$ Department of \\ General Science and Liberal Arts, King Mongkut's Institute of Technology, Ladkrabang Prince of Chumphon Campus, Chumphon 86160, \\ Thailand, ${ }^{3}$ BK21 plus, Department of Chemistry, Pusan National University, Busan 46241, ${ }^{4}$ Department of Biomedical Sciences, Dong-A \\ University, Busan 49315, Korea
}

Increased mRNA levels of cancer upregulated gene (CUG)2 have been detected in many different tumor tissues using Affymetrix microarray. Oncogenic capability of the CUG2 gene has been further reported. However, the mechanism by which CUG2 overexpression promotes cancer stem cell (CSC)-like phenotypes remains unknown. With recent studies showing that pyruvate kinase muscle 2 (PKM2) is overexpressed in clinical tissues from gastric, lung, and cervical cancer patients, we hypothesized that PKM2 might play an important role in CSC-like phenotypes caused by CUG2 overexpression. The present study revealed that PKM2 protein levels and translocation of PKM2 into the nucleus were enhanced in CUG2-overexpressing lung carcinoma A549 and immortalized bronchial BEAS-2B cells than in control cells. Expression levels of c-Myc, CyclinD1, and PKM2 were increased in CUG2-overexpressing cells than in control cells. Furthermore, EGFR and ERK inhibitors as well as suppression of Yap1 and NEK2 expression reduced PKM2 protein levels. Interestingly, knockdown of $\beta$-catenin expression failed to reduce PKM2 protein levels. Furthermore, reduction of PKM2 expression with its siRNA hindered CSC-like phenotypes such as faster wound healing, aggressive transwell migration, and increased size/number of sphere formation. The introduction of mutant S37A PKM2-green fluorescence protein (GFP) into cells without ability to move to the nucleus did not confer CSC-like phenotypes, whereas forced expression of wild-type PKM2 promoted such phenotypes. Overall, CUG2-induced increase in the expression of nuclear PKM2

*Corresponding author. Tel: +82-51-510-6116; Fax: +82-51-514-2358; E-mail: younghc@pusan.ac.kr

${ }^{\#}$ These authors contributed equally to this work.

https://doi.org/10.5483/BMBRep.2022.55.2.118

Received 23 August 2021, Revised 6 October 2021, Accepted 20 December 2021

Keywords: Cancer stem cell, Co-activator, CUG2, Non-metabolic function, PKM2 contributes to CSC-like phenotypes by upregulating c-Myc and CyclinD1 as a co-activator. [BMB Reports 2022; 55(2): 98-103]

\section{INTRODUCTION}

Using Affymetrix microarray system, increased CUG2 transcripts have been detected in many different cancer tissues including lung, ovary, and colon cancer tissues (1). CUG2's oncogenic activity is demonstrated by enhanced proliferation and tumor formation in nude mice (1). Moreover, capability of the CUG2 gene possesses CSC-like phenotypes in terms of rapid cell migration, an aggressive cell invasion, an enhanced sphere forming ability, and an increased doxorubicin-resistance through TGF- $\beta$ signaling $(2,3)$. These phenotypes have been linked to both EGFR/Stat1/HDAC4 signaling pathway ane $\beta$-catenin/yes-associated protein (Yap1)/NIMA-related kinase 2 (NEK2) signaling pathway $(4,5)$. According to recent studies, increased EGFR and $\beta$-catenin protein levels and signaling during CUG2 overexpression can be attributed to decreased Spry2 protein via c-Cbl (6).

In many cases, cancer cells display increased glucose consumption and lactate generation even under aerobic conditions, which are features of Warburg effect, also called aerobic glycolysis $(7,8)$. In this case, pyruvate kinase muscle 2 (PKM2), a key enzyme of glycolysis pathway, is responsible (7-9). According to further investigation, PKM2 protein which consists of four structural units (tetramer) has a high catalytic activity, resulting in high ATP production and more energetic catabolic metabolism $(7,10)$. Of interest, dimeric PKM2 protein (two structural units) displays a low catalytic activity, which provides glycolytic intermediates for anabolism $(7,10)$. PKM2 protein exists in the cytoplasm. It is translocated to the nucleus, suggesting that PKM2 has other functions $(8,9)$ in addition to glycolysis, such as acting as a protein kinase (11). For example, PKM2 protein does not use phosphoenolpyruvate as a metabolic substrate. Rather, PKM2 protein can take away of a phosphate group from phosphoenolpyruvate and then attach the phosphate group to Stat3

ISSN: 1976-670X (electronic edition)

Copyright (c) 2022 by the The Korean Society for Biochemistry and Molecular Biology

(c) This is an open-access article distributed under the terms of the Creative Commons Attribution Non-Commercial License (http://creativecommons.org/licenses/by-nc/4.0) which permits unrestricted non-commercial use, distribution, and reproduction in any medium, provided the original work is properly cited. 
(12), histone $\mathrm{H} 3$ (13), and ERK1/2 (14), allowing tumor cells to proliferate. As a co-activator, once translocated to the nucleus, PKM2 protein can bind to HIF-1 $\alpha$ protein (15). Eventually, the complex between PKM2 and HIF-1 $\alpha$ can accelerate mRNA synthesis of HIF-1 $\alpha$-targeting genes $(15,16)$. Furthermore, the protein complex between PKM2 and c-Src can be recruited into a CyclinD1 promoter containing $\beta$-catenin, leading to upregulation of CyclinD1 transcripts $(17,18)$.

This investigation was initiated to illustrate PKM2's role in CUG2-induced tumor development. Activities of both EGFR and ERK1/2 kinases contributed to increased PKM2 protein levels, leading to upregulation of c-Myc and CyclinD1 expression. Furthermore, Yap1 and NEK2 increased PKM2 protein expression, while $\beta$-catenin did not. Surprisingly, nuclear PKM2 was closely related to CSC-like phenotypes. Taken together, these findings suggest that PKM2 translocated into the nucleus possibly contributes to CSC-like phenotypes under CUG2 overexpression condition.

\section{RESULTS}

\section{CUG2 increases PKM2 protein levels and induces its} translocation to the nucleus

With recent studies documenting that PKM2 protein levels are increased in clinical tissues such as lung cancer (19), pancreatic ductal adenocarcinoma (20), colorectal cancer (21), and ovarian cancer tissues (22), we considered the possibility that PKM2 might contribute to CSC-like phenotypes induced by CUG2. Here, when PKM2 protein levels were compared between A549-CUG2

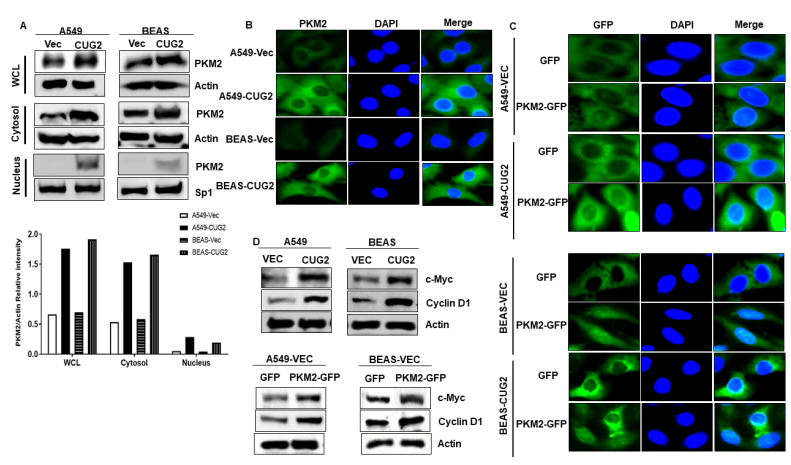

Fig. 1. Increased $P K M 2$ protein levels and $P K M 2$ translocation to the nucleus in CUG2-overexpressing cells. (A, D) Protein levels of PKM2, c-Myc, and CyclinD1 in the cytosol and nuclei or whole cell lysates from A549-CUG2, BEAS-CUG2 cell, and control cells were detected by Western blotting after 10\% SDS-polyacrylamide gel running. PKM2 expression under CUG2 overexpression was scanned and compared to that in control cells using Multi-Gauge Ver. 2.1 program. (B) For endogenous expression and localization of PKM2, cells were incubated with anti-PKM2 antibody followed by incubation with an Alexa Fluor488-conjugated secondary antibody. DNA was stained with DAPI. (C) WT GFP tagged-PKM2 expression was detected at $48 \mathrm{~h}$ after transfection using a fluorescence microscopy. As a control, GFP was also observed. and BEAS-CUG2 cells stably expressing CUG2 and the control cells stably expressing an empty vector, PKM2 protein levels were significantly higher in CUG2-overexpressed cells than in the control cells (Fig. 1A). Interestingly, when endogenous PKM2 was examined, the majority of PKM2 proteins were found in the cytosol, while certain portions of PKM2 were found in the nuclei of cells overexpressing CUG2 (Fig. 1B). Furthermore, when PKM2 was forcibly expressed by WT PKM2-GFP or GFP vector, GFP was found in the nuclei of cells transfected with WT PKM2-GFP vector. However, GFP was not detected in the nuclei of the cells transfected with GFP vector (Fig. 1C).

Furthermore, when we examined transcription factors that could be collaborators for nuclear PKM2 $(15,16)$, we discovered that CUG2 increases levels of c-Myc and CyclinD1 (Fig. 1D). Enforced expression of PKM2 also increased levels of these proto-oncogenic proteins (Fig. 1D). These results indicate that CUG2 can induce the upregulation of PKM2, leading to transferring of this protein into the nucleus and subsequently collaborating with proto-oncogenic proteins such as c-Myc and CyclinD1.

\section{Both EGFR-ERK and Yap1-NEK 2 signaling transduction increase PKM2 expression}

As previously reported, both EGFR-ERK and the $\beta$-catenin-NEK2 signaling transduction via Yap1 are closely related to CSC-like phenotypes mediated by CUG2 $(4,5)$. As a result, we considered the possibility that these signaling pathways might contribute to the increase in PKM2 expression. Once EGFR and ERK inhibitors were administrated under CUG2 overexpression condition, reduced levels of PKM2 protein were notified (Fig. 2A). Lower phosphorylation levels of target proteins also confirmed that these inhibitors were working (Fig. 2A). Furthermore, lower PKM2 protein levels were detected in both cytosol and nucleus after treatment with EGFR and ERK inhibitors compared with those after treatment with DMSO, confirming that the EGFR-ERK signaling axis was involved in the expression of PKM2 (Fig. 2A). After $\beta$-catenin, Yap1, and NEK2 were suppressed with attendant siRNA, PKM2 protein levels were examined.



Fig. 2. Elevated PKM expression is attributed to upregulation of EGFRERK and Yap1-NEK2 signaling. (A, B) Kinase activities of EGFR and ERK1/2 and protein expression levels of Yap1 and NEK2 were measured by Western blotting after gefitinib $(20 \mu \mathrm{M})$ or PD98059 $(30 \mu \mathrm{M})$ treatment and administration of Yap1 or NEK2 siRNA (200 ng). 
We found that PKM2 protein levels were reduced during Yap1 and NEK2 suppression. However, $\beta$-catenin suppression did not reduce PKM2 protein expression (Fig. 2B). Therefore, we propose that elevated PKM2 protein levels not only can be caused by increased kinase activity of EGFR and ERK $1 / 2$, but also can be caused by enhanced expression of Yap1 and NEK2.

\section{CSC-like phenotypes are attributed to PKM2 protein under CUG2 overexpression}

We wondered whether PKM2 might play an important role in CSC-like phenotypes such as rapid cell migration, assertive invasion, enhanced ability of sphere formation, and increased resistance to anti-cancer drug under CUG2 overexpression because we discovered increased PKM2 protein levels under CUG2 overexpression in previous tests (Fig. 1A). To answer this question, when PKM2 protein levels were suppressed with attendant siRNA, CUG2-induced CSC-like phenotypes were explored. In this case, PKM2 suppression resulted in slower migration (Fig. 3A) and invasion (Fig. 3B) than control siRNA treatment. Furthermore, PKM2 knockdown reduced both the number and size of spheroids (Fig. 3C). In addition, our previous studies have documented that under CUG2 overexpression, resistance to doxorubicin-induced cytotoxicity is closely related to a decrease of reactive oxygen species production (23). Herein, we observed drastically enhanced reactive oxygen species (ROS) production and cleaved PARP levels in cells during PKM2 knockdown, indicating increased sensitivity of

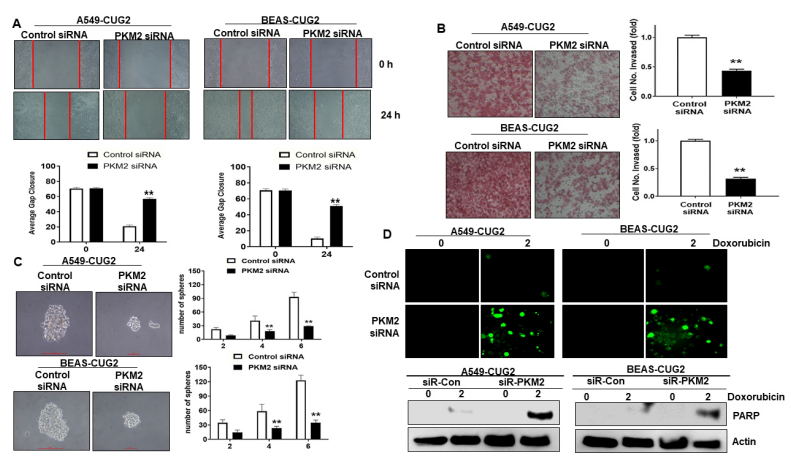

Fig. 3. Treatment with PKM2 siRNA diminishes CUG2-mediated CSC-like phenotypes. (A) Migrated cells filled in gaps scratched with a $200 \mu \mathrm{l}$ tip after transfection with PKM2 siRNA. Migration of cells transfected with PKM siRNA was compared with that of cells treated with control siRNA. (B) As cells treated with PKM2 siRNA invaded from upper to lower wells, the number of cells in the lower wells was counted after hematoxylin-eosin staining. **P $<0.01$, PKM2 siRNA vs. control siRNA. (C) PKM2 siRNA or control siRNA was added to cells. The size and number of spheres were measured over six days. A sphere with a diameter greater than $50 \mu \mathrm{m}$ was a criterion for sphere formation. **P $<0.01$, PKM2 siRNA vs. control siRNA. (D) After transfection with PKM2 siRNA or control siRNA, cells were treated with doxorubicin for $12 \mathrm{~h}$. ROS levels in cells were detected using $20 \mu \mathrm{M}$ of DCF under a fluorescence microscope. Apoptosis was measured by immunoblotting using anti-cleaved PARP antibodies. apoptosis to doxorubicin (Fig. 3D). These results imply that CSC-like phenotypes are attributed to PKM2 protein under CUG2 overexpression.

\section{Nuclear PKM2 during enforced expression contributes to CSC-like phenotypes}

Because there are numerous lines of evidence showing that PKM2 protein performs non-metabolic functions in tumor formation $(11,15-18)$, we focused on PKM2's role as a co-activator. As a result, we introduced mutant Ser37A PKM2-GFP that could not be translocated to the nucleus during EGF stimulation (14). We also observed that forced expression of mutant Ser37A PKM2-GFP failed to show PKM2 localization in cell nuclei (Fig. 4A). To verify that ERK could phosphorylate PKM2 at Ser37 as described previously (14), ERK inhibitor was used to treat A549-CUG2 and BEAS-CUG2 cells. Phosphorylation levels of PKM2 at Ser37 were then examined using a specific phospho-Ser37-PKM2 antibody. We found that treatment with ERK inhibitor reduced phosphorylation levels of PKM2 at Ser37 (Fig. 4A).

In comparison with WT PKM2-GFP transfection, mutant Ser37A PKM2-GFP transfection resulted in slower migration and invasion of control cells (Fig. 4B, C). Furthermore, mutant expression of

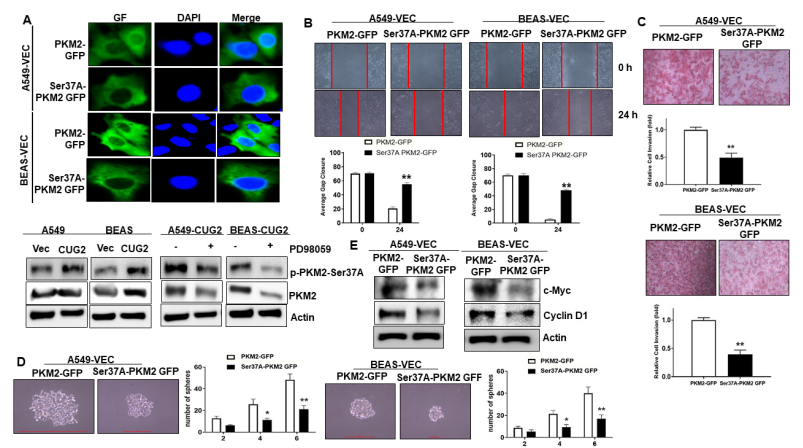

Fig. 4. Translocalization of PKM2 to the nucleus contributes to generation of CSC-like phenotypes. (A) Immunofluorescence was used to examine the localization of PKM2 in transfected cells at $36 \mathrm{~h}$ post transfection of cells with WT PKM2-GFP $(2 \mu \mathrm{g})$ or mutant Ser37A PKM2-GFP ( $2 \mu \mathrm{g})$. DNA was stained with DAPI. After treatment with ERK inhibitor, phosphorylation levels of PKM2 at Ser37 were detected with a corresponding antibody. (B) After transfection of cells with WT PKM2-GFP (2 $\mu \mathrm{g}$ ) or mutant Ser37A PKM2-GFP $(2 \mu \mathrm{g})$, gap closure areas were measured for cell migration under a phase-contrast microscope $(100 \times$ magnification). (C) Cells treated with WT PKM2-GFP or mutant Ser37A PKM2-GFP invaded from upper to lower wells and the number of cells in the lower wells was counted afer haematoxylin-eosin staining. $* * \mathrm{P}<0.01$, WT PKM2-GFP vs. mutant Ser37A PKM2-GFP. (D) After transfection of cells with WT PKM2-GFP or mutant Ser37A PKM2-GFP, the size and number of spheroids were measured and counted over six days. A spheroid exceeding $50 \mu \mathrm{m}$ was a criterion for sphere formation. ${ }^{*} P<0.05$, WT PKM2-GFP vs. mutant Ser37A PKM2-GFP; $* * \mathrm{P}<0.01$, WT PKM2-GFP vs. mutant Ser37A PKM2-GFP. (E) Protein expression of CyclinD1 and c-Myc after transfection with WT PKM2-GFP or mutant Ser37A PKM2-GFP was examined by Western blotting. 
PKM2 exhibited a lower number of smaller-sized spheroids in cells than WT PKM2-GFP (Fig. 4D). Moreover, enforced expression of mutant Ser37A PKM2-GFP induced lower NEK2 kinase activity and $\beta$-catenin transcriptional activity than WT PKM2-GFP transfection (Supplementary Fig. 1). Furthermore, WT PKM2-GFP transfection resulted in higher levels of c-Myc and CyclinD1 protein than mutant PKM2 transfection (Fig. 4E). These findings suggest that PKM2 might have a CSC-like capacity as a co-activator when it is combined with proto-oncogenic proteins such as c-Myc and CyclinD1.

\section{DISCUSSION}

The Warburg effect, a common marker of tumor cell metabolism, is characterized by cancers' increased utilization of glucose and increased production of lactate via the glycolysis pathway even under normal conditions ( $20 \%$ oxygen concentration) $(7$, 8). It has been reported that the Warburg effect is attributed to behaviors of PKM2 protein (9). Based on these lines of evidence (7-9), we performed nuclear magnetic resonance spectroscopy analysis for CUG2-overexpressing cells and control cells to determine their differences in glycolytic metabolites and amino acids. Lactate production was not significantly different between A549-CUG2 and A549-Vec cells. However, BEAS-Vec produced more lactate than BEAS-CUG2 cells (Supplementary Fig. 2). Because there were no significant differences in other metabolites between the two, we hypothesized that PKM2 could not function as a metabolic enzyme in the aerobic glycolytic pathway.

Because we observed that PKM2 protein was translocated to the nucleus, we considered the idea that PKM2 protein might act as a co-activator. Previous research has shown that translocated PKM2 proteins can bind to Jumonji-C-domain-containing dioxygenase Jumonji-containing protein 5 in the nucleus and promote HIF-1 $\alpha$ expression (16). Other studies have also shown that the interaction between PKM2 and c-Src is required for the recruitment to CyclinD1 promoter during EGFR signaling (17). Another study has reported that nuclear PKM2 protein can act as a co-activator together with $\beta$-catenin to express c-Myc (18). These lines of evidence support the translocation of PKM2 to the nucleus in the presence of CUG2 overexpression as well as increased expression of CyclinD1 and c-Myc in the presence of PKM2 as confirmed by PKM2 siRNA treatment and transfection with mutant Ser37A PKM2-GFP (unable to translocate into the nucleus). Since PKM2 phosphorylated histone $\mathrm{H} 3$ can act as a protein kinase which contributes to the development of tumors (13), our findings suggest that PKM2 can induce CSC-like phenotypes such as a speedy cell migration, assertive invasion, and increased spheroid size and number, which do not rule out a protein kinase role of PKM2.

Therefore, PKM2 protein has been considered a potential target for cancer therapy. Treatment with Shikonin, a natural compound derived from Lithospermum erthrorhizon, could suppress glycolytic rate that is determined by lactate production and glucose consumption (24). It could also inhibit 12-O-tetra- decanoylphorbol 13-acetate (TPA)-induced cellular transformation and activation of PKM2 (25). Administration of Selumetinib, a miteogen-activated protein kinase kinase inhibitor, can diminish phosphorylation of ERK and prevent EGF-induced nuclear translocation of PKM2, ultimately suppressing tumor cell growth (14). On the other hand, FFJ-5 and FFJ-3 as structurally modified mollugins could attenuate PKM2 expression through EGFRAkt-PKM2 and PI3K-Akt signaling pathways, respectively (26) (27). These lines of evidence support our finding that PKM2 has an oncogenic role under CUG2 overexpression condition.

\section{MATERIALS AND METHODS}

\section{Antibodies and reagents}

Cell Signaling Biotechnology (Danvers, MA, USA) provided antibodies that target $\beta$-catenin, EGFR, and ERK $1 / 2$, as well as phospho- $\beta$-catenin, phospho-EGFR, phospho-ERK1/2, and PKM2. Antibody recognizing phospho-PKM2(Ser37) was purchased at Thermo Fisher Scientific (Walltham, MA, USA). Anti- Yap1 and -NEK2 antibodies were acquired from Abcam (Cambridge, MA, USA). Furthermore, Santa Cruz Biotechnology (Santa Cruz, CA, USA) provided antibodies against CyclinD1 and c-Myc obtained. Finally, gefitinib and PD98059 purchased from Calbiochem (San Diego, CA, USA) was used to inhibit EGFR and ERK1/2 kinase activity, respectively.

\section{Cell culture and transfection}

A549 and BEAS-2B cells purchased from ATCC (Manassas, VA, USA) were stably introduced with wild type (22) CUG2 vector, while the control cells were treated with an empty vector with the same construct. A549-CUG2 and -Vec cells were maintained in RPMI-1640 whereas BEAS-CUG2 and -Vec cells were kept in DMEM medium. The media additionally contained $10 \%$ FBS, $1 \%$ penicillin, $1 \%$ streptomycin, and G418 $(500 \mu \mathrm{g} / \mathrm{ml}$; Sigma Aldrich, St. Louis, $\mathrm{MO}$, USA) at $37^{\circ} \mathrm{C}$ and $5 \% \mathrm{CO}_{2}$. After reaching a confluence of $50 \%-60 \%$, the cells were transfected with siRNAs (Bioneer, Daejeon, Korea) targeting PKM2, $\beta$-catenin, Yap1, and NEK2 using Lipofectamine ${ }^{\circledR} 2000$ (Thermo Fisher Scientific, Carlsbad, CA, USA). The same protocol was applied for the transfection of the cells with wild type (22) PKM2- GFP (Addgene, Watertown, MA, USA) or mutant Ser37A PKM2-GFP vector generated by Bioneer.

\section{Cellular fractionation}

Cellular fractionation was performed as previously mentioned (2). The supernatants were obtained as the soluble fractions, after the cells were treated with a TTN buffer including $0.05 \%$ Triton X-100 and centrifuged. Then, the pellets were treated with a RIPA buffer including $0.1 \%$ SDS, $1 \%$ NP-40, and $0.5 \%$ deoxycholic acid, and followed by centrifugation. The nuclear extracts were thereafter obtained.

\section{Wound healing assay}

The cells were cultured until they were approximately $80 \%$ 
confluent. The gap was measured by observing the cells under a microscope for $24 \mathrm{~h}$.

\section{Transwell invasion assay}

Transwell invasion assay was carried out as formerly mentioned $(2,3)$. Briefly the cells in the upper well invaded the lower wells containing serum through the coated membrane (BD biosciences). After fixing and staining with hematoxylineosin, the images were obtained then under a microscopy (100× magnification). The experiment was tested in triplicates. The data designate the mean + the standard deviations (SD).

\section{Sphere forming assay}

For six days, the cells treated with expression vectors or siRNAs were incubated in a medium containing $0.4 \%$ BSA, EGF (20 $\mathrm{ng} / \mathrm{ml})$, basic-FGF $(10 \mathrm{ng} / \mathrm{ml})$, and insulin $(5 \mu \mathrm{g} / \mathrm{ml})$. The spheroids in wells with ultra-low attachment were measured and counted under an optical microscope. The assay was tested in triplicates. The data designate the mean + the SD.

\section{Western blotting}

Western blotting was performed as previously explained $(2,3)$. Proteins were segregated from cell lysates using a 10\% SDSpolyacrylamide gel and the gel was then conveyed into nitrocellulose membranes. The membrane was then incubated primary antibodies (1:500-1,000 dilution) and subsequently a horseradish peroxidase-conjugated secondary antibody. The images were obtained after treatment with a peroxidase substrate (Thermo Fisher Scientific).

\section{Immunofluorescence microscopy}

PKM2 protein expression and localization were investigated using immunofluorescence as previously described (2). Anti-PKM2 antibody was added to the cells fixed and permeabilized. Thereafter, the treated cells were incubated with Alexa Fluor 488conjugated secondary antibody for $1 \mathrm{~h}$. After 4',6-diamidino-2phenylindole (DAPI) was added to the cells for staining the nuclei, the images were subsequently visualized with a fluorescence microscope.

\section{Measurement of reactive oxygen species}

The intracellular reactive oxygen species (ROS) levels were measured using a fluorescence microscope after treatment with 20 $\mu \mathrm{M}, 2^{\prime}, 7^{\prime}$-dichlorodihydrofluorescein diacetate (DCF; Molecular Probes, Eugene, OR, USA) for $30 \mathrm{~min}$.

\section{Statistical analysis}

All data are presented in terms of mean \pm SD. For comparison between two groups, the results were analyzed using Student's unpaired t-test. When the P-value is greater than 0.05 , the data is considered significant.

\section{ACKNOWLEDGEMENTS}

This study was supported by the National Research Foundation of Korea (NRF) grant funded by the Korean government (MSIT) (2020R 1F1A 1048183) and the Technology Innovation Program (N0002310 and P0008763) funded by the Ministry of Trade, 511 Industry, and Energy (MOTIE, Korea). This work was also supported by Korea Institute for Advancement of Technology (KIAT) grant funded by the Korea Government (MOTIE) (P0008763, The Competency Development Program for Industry Specialist).

\section{CONFLICTS OF INTEREST}

The authors have no conflicting interests.

\section{SUPPLEMENTARY DATA}

Supplementary data to this article can be found online.

\section{REFERENCES}

1. Lee S, Gang J, Jeon SB et al (2007) Molecular cloning and functional analysis of a novel oncogene, cancer-upregulated gene 2 (CUG2). Biochem Biophys Res Commun 360, 633-639

2. Kaowinn S, Kim J, Lee J et al (2017) Cancer upregulated gene 2 induces epithelial-mesenchymal transition of human lung cancer cells via TGF- $\beta$ signaling. Oncotarget 8, 5092

3. Kaowinn S, Seo EJ, Heo W et al (2019) Cancer upregulated gene 2 (CUG2), a novel oncogene, promotes stemness-like properties via the NPM1-TGF- $\beta$ signaling axis. Biochem Biophys Res Commun 514, 1278-1284

4. Kaowinn S, Kaewpiboon C, Koh SS, Krämer OH and Chung YH (2018) STAT1 HDAC4 signaling induces epithelial mesenchymal transition and sphere formation of cancer cells overexpressing the oncogene, CUG2. Oncol Rep 40, 2619-2627

5. Kaowinn S, Yawut N, Koh SS and Chung YH (2019) Cancer upregulated gene (CUG) 2 elevates YAP1 expression, leading to enhancement of epithelial-mesenchymal transition in human lung cancer cells. Biochem Biophys Res Commun $511,122-128$

6. Yawut N, Kaewpiboon C, Budluang P et al (2020) Overexpression of cancer upregulated gene 2 (CUG2) decreases Spry2 through c-Cbl, leading to activation of EGFR and $\beta$-catenin signaling. Cancer Manag Res 12, 10243

7. Hsu PP and Sabatini DM (2008) Cancer cell metabolism: Warburg and beyond. Cell 134, 703-707

8. Dong G, Mao Q, Xia W et al (2016) PKM2 and cancer: The function of PKM2 beyond glycolysis. Oncol Lett 11, 1980-1986

9. Dayton TL, Jacks T and Vander Heiden MG (2016) PKM 2, cancer metabolism, and the road ahead. EMBO Rep 17, $1721-1730$

10. Whitfield JR and Soucek L (2012) Tumor microenvironment: becoming sick of Myc. Cell Mol Life Sci 69, 931-934 
11. Gao X, Wang H, Yang JJ, Liu X and Liu ZR (2012) Pyruvate kinase $M 2$ regulates gene transcription by acting as a protein kinase. Mol Cell 45, 598-609

12. Yang P, Li Z, Fu R, Wu H and Li Z (2014) Pyruvate kinase M2 facilitates colon cancer cell migration via the modulation of STAT3 signalling. Cell Signal 26, 1853-1862

13. Gernapudi R, Yao Y, Zhang Y et al (2015) Targeting exosomes from preadipocytes inhibits preadipocyte to cancer stem cell signaling in early-stage breast cancer. Breast Cancer Res Treat 150, 685-695

14. Yang W, Zheng Y, Xia Y et al (2012) ERK1/2-dependent phosphorylation and nuclear translocation of PKM2 promotes the Warburg effect. Nat Cell Biol 14, 1295-1304

15. Luo W, Hu H, Chang R et al (2011) Pyruvate kinase M2 is a PHD3-stimulated coactivator for hypoxia-inducible factor 1. Cell 145, 732-744

16. Wang HJ, Hsieh YJ, Cheng WC et al (2014) JMJD5 regulates PKM2 nuclear translocation and reprograms HIF-1 $\alpha^{-}$ mediated glucose metabolism. Proc Natl Acad Sci U S A $111,279-284$

17. Yang $\mathrm{W}, \mathrm{Xia}$ Y, Ji $\mathrm{H}$ et al (2011) Nuclear PKM2 regulates $\beta$-catenin transactivation upon EGFR activation. Nature 480, 118-122

18. Wu H, Li Z, Yang P, Zhang L, Fan Y and Li Z (2014) PKM2 depletion induces the compensation of glutaminolysis through $\beta$-catenin/c-Myc pathway in tumor cells. Cell Signal 26, 2397-2405

19. Wang C, Zhang S, Liu J et al (2020) Secreted pyruvate kinase M2 promotes lung cancer metastasis through activating the integrin Beta1/FAK signaling pathway. Cell Rep 30, 1780-1797. e6
20. Xu Q, Wu N, Li X et al (2019) Inhibition of PTP1B blocks pancreatic cancer progression by targeting the PKM2/AMPK/ mTOC1 pathway. Cell Death Dis 10, 1-15

21. Bian Z, Zhang J, Li M et al (2018) LncRNA-FEZF1-AS1 promotes tumor proliferation and metastasis in colorectal Cancer by regulating PKM2 signaling. Clin Cancer Res 24, 4808-4819

22. Ahmed A, Dew T, Lawton F et al (2007) M2-PK as a novel marker in ovarian cancer. A prospective cohort study. Eur J Gynaecol Oncol 28, 83-88

23. Kaowinn S, Jun SW, Kim CS et al (2017) Increased EGFR expression induced by a novel oncogene, CUG2, confers resistance to doxorubicin through Stat1-HDAC4 signaling. Cell Oncol 40, 549-561

24. Chen J, Xie J, Jiang Z, Wang B, Wang Y and Hu X (2011) Shikonin and its analogs inhibit cancer cell glycolysis by targeting tumor pyruvate kinase-M2. Oncogene 30, 42974306

25. Li W, Liu J and Zhao Y (2014) PKM2 inhibitor shikonin suppresses TPA-induced mitochondrial malfunction and proliferation of skin epidermal JB6 cells. Mol Carcinog 53, 403-412

26. Wei X, Li M, Ma M et al (2017) Induction of apoptosis by FFJ 5, a novel naphthoquinone compound, occurs via downregulation of PKM2 in A549 and HepG2 cells. Oncol Lett 13, 791-799

27. Li D, Wei X, Ma M et al (2017) FFJ 3 inhibits PKM2 protein expression via the PI3K/Akt signaling pathway and activates the mitochondrial apoptosis signaling pathway in human cancer cells. Oncol Lett 13, 2607-2614 